\title{
Ueber die festen Kohlenstoffverbindungen in Meteoriten ${ }^{*}$ ); \\ von J. Laurence Smith, Louisville, Ky.
}

\section{Kohlenmetsorite.}

Gewisse Meteorite werden wegen ihres Aussehens und wegen eines, wenn auch geringen Gehaltes an Kohlenstoff Kohlenmeteorite genannt. Man kennt deren nur vier, nämlich Alais, gefallen 1806, Kold-Bokeveldt, gefallen 1838, Kaba, 1857, und Orgueil, 1864 gefallen. Ihr Gehalt an kohliger Substanz beträgt resp. etwa $3,2,0,6$ und 6 pC. Alais, KoldBokeveldt und Orgueil stehen sich unter einander näher, als dem Kabameteorit. Die hauptsächlichsten Mineralbestandtheile sind etwa folgende :

$\begin{array}{lcccc} & \text { Alais } & \text { K-Bok. } & \text { Kaba } & \text { Orgueil } \\ & \text { Berzelius } & \text { Harris } & \text { Wöhler } & \text { Pisani } \\ \text { Kieselsłure } & 31,22 & 30,80 & 34,24 & 26,08 \\ \text { Magnesis } & 22,21 & 22,20 & 22,39 & 17,00 \\ \text { Eisenoxydul } & 29,03 & 29,94 & 26,20 & 29,60 .\end{array}$

Vergleicht mar damit die Hauptbestandtheile einiger genau untersuchten Steinmeteorite :

$\begin{array}{lcccccc} & \text { Chassigny } & \begin{array}{c}\text { Chatesu } \\ \text { Renard }\end{array} & \begin{array}{c}\text { Harrison } \\ \text { County }\end{array} & \text { Concord } & \text { Danville } & \begin{array}{c}\text { Sears- } \\ \text { mont }\end{array} \\ \text { Kieselsáure } & 35,30 & 38,13 & 47,06 & 47,80 & 50,08 & \mathbf{4 0 , 6 1} \\ \text { Magnesia } & 31,76 & 17,67 & 27,61 & 24,53 & 20,14 & \mathbf{8 6 , 3 4} \\ \text { Eisenoxydul } & 26,70 & 29,44 & 26,05 & 28,03 & 19,85 & 19,21\end{array}$

so ergiebt sich die höclist überraschende und häufig übersehene Thatsache, dafs die Zusammensetzung der schwarzen Meteorite ganz dem gemeinsamen Typus der Steinmeteorite entspricht. In beiden Arten von Meteoriten haben wir die

*) Vom Verfaseer mitgetheilt.

Annalon der Chemie 182. Bd. 


\section{Smith, über die festen Kohlenstoffverbindungen}

gleichen Mineralien, nämlich Olivin und Pyroxen; sie unterscheiden sich nur durch die melir oder weniger dichte Beschaffenheit dieser Mineralien.

Von mancher Seite wird die erwähnte Thatsache nicht genügend gewürdigt. So spricht 7. B. Neunier in einer Abhandlung über den Ursprung der Meteorite *) sein Ẹrstaunen daruber aus, dafs ich von einer bis zu cinem gewissen Grade gleichmäfsigen Zusammensetzung drr Meteorite rede und daraus einen Beweis für die Gemeinsamkeit ihres kosmischen Ursprungs herleite. $\mathrm{Er}_{\boldsymbol{r}}$ ist so ganz entgegengesetzter Ansicht, dafs er behauptet, zwischen den Eisenmeteoriten, den Olivin-, Thon- und Kohlenmeteoriten beständen eben so grofse Unterschiede, wie zwischen den verschiedenartigsten terrestrischen Gesteinen; eine Behauptung, welche also alle Felsarten und sedimentären Gehilde vom Basalt und Granit bis zur Kreide und den Tertiarablagerungen einschliefsen wärde.

Niemand wird in obiger Zusammenstellung eine solche Verschiedenheit erkennen und doch umschliefst dieselbe die zwei in ihren äufseren Eigenschaften extremeu Repräsentanten von Meteoriten. Es ist zur Genüge dargethan, dafs drei oder vier Mineralien, Nickeleisen, Olivin, Pyroxen, Anorthit, hauptsächlich die drei ersteren, in wechseindem Verfältnifs die Hauptmasse eines jeden Meteorits zusammensetzen und die reinen Eisenmeteorite sind zuletzt nur gröfsere Massen der metallischen Partikeln, welche in jedem Meteorit, die Kohlenmeteorite nicht ausgenommen, gefunden werden *).

*) Cosmos, Dechr. 1869.

**) Orgueil und Rhoda sind die einzigen Meteorite, in welchen Nickelelsen bis jetzt noch nicht mit Bostimmtheit nachgewiesen wurde; im Orgueil wurden übrigens nahezu $3 \mathrm{pC}$. Nickel- und Kobaltoxydul gefunden; Rhode ist noch nicht ganz genau untersucht. 
Es ist nicht meine Absicht, hier die allgemeine Aehnlichkeit in der Zusammensetzung der Meteorite des Weiteren zu besprechen; ich wollte nur constatiren, dafs die Kohlenmeteorite trotz ihrer schwarzen Farbe und pulverigen Beschaffenbeit sich in ihren Mineralbestandtheilen von den 80genannten gewöhnlichen Meteoriten nicht wesentlic! unterscheiden; wir werden sehen, dafs sie durch ihren kohlenstoffhalligen Bestandtheil sich aufs engste auch an die Fisenmeteorite anschliefsen.

\section{Oraphit aus Eisenmeteoriten.}

Beim Durchschneiden lat man bekanntlich im Inneren der Eisenmeteorite verschiedentliche knollige Concretionen gefunden. Dicselben bestehen am häufigsten aus Eisensulfür oder Troïlit und bilden die Ausfüllung ovaler Hohlräume. Manchmal sind diese Troilite mit einer dünnen und helleren Schicht von Schreibersit überzogen und letzterer kommt auch allein in eckigen oder blätterigen Concretionen vor.

Seltener werden Conoretionen beobachtet, welche aus Kohlenstoff von graphitischer Natur bestehen; wie der Trö̈lit. bilden sie gewöhnlich die Ausfüllung unregelmälsig eiförmiger Hohlräume; sie sind melı oder weniger mit Trö̈lit vermengt.

Von solchen Eisenmeteoriten mit Graphiteinlagerung hatte ich hauptsâchlich vier unter Händen : Toluca, Cranbourne, De Kalb und Sevier; die beiden letzteren habe ich genauer untersucht. Im Aussehen sind die Graphitknollen mehr oder weniger verschieden, obwohl sie sich sonst ganz gleich verhalten. Das Hauptmaterial für meine Untersuchung lieferte ein gerade aus dem Mittelpunkt des Seviermeteorits entnommener Graphitknollen, der gröfste, den ich je gesehen und vielleicht der gröfste, der überhaupt gefunden wurde. Es gelang, denselben ganz und völlig unverletzt von dem Eisen loszulüsen. Er war 60 MM. lang und 20 bis 35 MM. 
dick und wog vor dem Durchschneiden $92 \mathrm{Grm}$; von Gestalt war er unregelmäfsig, Handeln ăhnlich, auf einer Seite abgeplattet, von etwas höckeriger Oberfläche. Die Farbe ist graphitschwarz, mit Ausnahme einzelner Stellen, an denen kleine bronzefarbene Troilite aufsitzen. $\mathrm{Er}$ ist von dichtem und compactem Gefüge; die Sãge durchschneidet inn leicht, nur hier und da begegnet sie härteren Troilitpartikeln. Nach Structur und Pulver ist er dem dichten Graphit von Borrowdale in Cumberland ähnlich und gänzlich verschieden von dem schuppigen Graphit von Ceylon oder dem Hochofengraphit.

Am Durchschnitt wurde Folgendes beobachtet : Ungefähr ein Funftel des Umfangs wird von Troilit in etwa 1 MM. dicker Schicht gebildct; die ganze übrige Schnittläche zeigt bis auf wenige Stellen ganz das Aussehen des Graphils. Im Inneren fand sich ein kleiner Kern von Troilit, in der Form dem ganzen Kmollon abnlich, yon ungefäh 10 zu $5 \mathrm{MM}$. Durchmesser und durch einen zwischengelagerten Graphitgürtel von etwa $1 / 2$ bis 1 MM. Dicke in zwei concentrische Lagen geschichtet.

Das specifische Gewichl wurde $=2,26$ gefunden. Zur Bestimmung diente ein anscheinend von Troïlit völlig freies Stück; vor der Wägung in Wasser war die Luft aus den Poren mittelst der Luftpumpe entfernt worden.

\section{Chemisches Verhalten des Meteorgraphits.}

Erhizzt wan den gepulverten Graphit in Glasrōhrchen auf 100 bis $150^{\circ}$, so entweicht das hygroscopische Wasser; erhitzt man ein wenig stärker und bringt die Substanz nahe unter die Nase, so nimmt man einen schwach empyreumatischen Geruch wahr: bei noch stärkerem Erhitzen macht sich schwacher Schwefilwasserstoffgeruch bemerkbar. An der Luft 
erhitzt verbrennt die Kohle nur sehr schwierig, wodurch sich ihre graphitische Natur kennzeichnet.

Aus $2 \mathrm{Grm}$. des Graphits wurde durch Anreiben mit Aether und längere Digestion bei gewöhnlicher Temperatur ein àtherischer Auszug bereitet. Dieser wurde im offenen Becherglas bei etwa $3^{\circ}$ verdunstet. Nach dem Verdunsten des Aethers fand man die $W$ and des Glases bedeckt von langen farblosen nadelförmigen Kryställchen, kürzere safsen auf dem Boden. Aufserden waren einige rhombenförmige Kryställchen und rundliche Körnchen bemerkbar. Der Rückstand hatte einen eigenthümlich aromatischen, etwas an Lauch erinnernden Geruch. Seine Menge war gering; sie betrug nicht über $15 \mathrm{Mgrm}$. aus $2 \mathrm{Grm}$. Substanz. Auf dem Platinblech erhitzt schmelzen die Kryställchen bei etwa $120^{\circ}$. Im Glasröhrchen schmelzen sie erst, dann verflüchtigen sie sich unter Hinterlassung eines kohligen Rückstandes. Die Dämpfe verdichten sich zu gelben Tropfen, welche rasch erstarren. In Alkohol sind die Krystalle nicht löslich, leicht in Schwefelkohlenstoff. Durch rauchende Salpetersäure werden sie oxydirt, die Losung enthält Schwefelsäure. Für eine Elementaranalyse war die Menge zu gering, doch liefs sich constatiren, dafs Schwefel den Haupthestandtheil bildet; der Rest besteht aus Kohlenstoff und Wasserstoff. Der Körper ist daher entweder ein eigenthümlicher Sulfokohlenwasserstoff (in einer früheren Notiz habe ich diese Verbindung Celestialit genannt), oder er ist Schwefel nit einer kleinen Menge von Kohlenwasserstoff vermengt, welche dell eigenthümlichen Geruch und die abweichende Krystallform bedingt.

Das mit Aether erschöpte Graphitpulver wurde nun mit Sckwefelkohlenstoff behandelt. Beim Verdunsten der Lösung hinterblieb eine kleine Menge eines festen gelben Körpers, der nach Geruch und Verhalten beim Erhitzen mil dem Abdampfrückstand der ätherischen Lösung identisch erschien. 


\section{Smith, über die festen Kohlenstoffverbindungen}

Aehnliche Resultate lieferten die Graphite aus dem De Kalb und Cranbourne.

Finwirkung von concentrirter Salpetersäure auf Meteorgraphit. - Nach dem Auszichen mil Aether und Schwefelkohlenstoff wurde der Graphit mit starker Sulpetersäuro lw:handelt. Unter heftiger Reaction löst dicse das eingespreng:t: Schwofeleisen. Nach längerer Digestion wurde filtrirt, der Rückstand wohl ausgewaschen und getrocknet; er botruir $55 \mathrm{pC}$. der ursprünglichen Substanz und bestand hauptsächlich aus Kohlenstoff. An der Luft verbrennt derselbe sehr scliwicrig, leicht dagegen im Sauerstoff; er hinterliefs $1 \mathrm{pC}$. Asche.

Die Salpetersäurelōsung wurde analysirt, sie enthiclt die Elemente d:s Troilits :

$\begin{array}{lr}\text { Schwefel } & 35,05 \\ \text { Eisen } & 62,21 \\ \text { Kobalt } & 0,56 \\ \text { Nickel } & 0,16 \\ \text { Magnesium } & 0,30 \\ \text { Kieselsüure } & 0,21 \\$\cline { 2 - 2 } & $\left.\frac{98,49}{}\end{array}\right\}$ Troülit.

Es ist bemerkenswerlh, dafs dieses Schwefeleisen so wenig Nickel und Kobalt enthält, da es durch Absonderung aus der Meteoreisenmasse entstanden seil muls; weiter mufs es auffallen, dafs darin Kobalt gegen Nickel vorherrsilit, während doch in der ungebenden Eisenmasst umgekehrt das Nickel gegenüber dem Kobalt weit überwiegt. Doch möclite ich diers Verbaltnifs nicht als allgemeine Regel hinstellen, ehe es an einer gröfseren Zahl von Fällen Bestätigung ge:funden hat.

Einwirkung einer Mischung von chlorsaurem Kali und rauchender Salpetersäure. - Mittelst dieses Oxydationsgemisches stellte bekanntlich Brodie *) seine Graphitsäure

*; Jieso Annalen 119. 9. 
dar. Später fand Berthelol*), dafs sich die verschiedenen Kohlemodificationen gegen das Gemisch verschieden verhalten und dafs nur die eigentlichen Graphite jene Verbindung liefern, die er Graphitoxyd nannte. Berthelot untersuchte auch einige meteorische Graphite; aus Cranbourne erhielt er Graphitoxyd, aus der kohleartigen Suhstanz des Orgueilmeteoriten aber nicht.

Ich habe in gleicher Weise die meteorischen Graphite aus Sevier, De Kalb und Cranbourne untersucht; auch die kohleartige Materie des Orgueil habe ich von Neuem auf ihr Verhalten gegen jenes Oxydationsgemisch geprüft.

Ich verfuhr ganz nach den Angaben Berthelot's. Die fein gepulverte Substanz wurde durch starke Salpetersäure von Schwefeleisen befreit, ausgewaschen, getrocknet und mit dem füflachen Gewicht chlorsauren Kali's vermengt. Diese Mischung wurde dann in kleinen Portionen allmälig in so viel rauchende Salpetersäure eingetragen, als nöthig war, die Masse völlig zu durchfeuchten; zur Verhütung einer Explosion wurde die Schale mit Salpetersäure in Eiswasser gestellt. Nach einigen Stuaden wurde die Mischung in einen Kollen gegeleen und im Wasserbade einige Tage lang bei 50 bis $60^{\text {st }}$ rwarmt. Sevier- und De Kalbgraphit lieferten hierbei Craphitoxyd mit allen Eigenschaften, wie sie Berthelot von dem Graphitoxvd aus Cranbournegraphit angegeben hat; aus letzterem habe auch ich das Oxyd dargestellt.

Die Meteorgraphite oxydiren sich weit rascher als irgend ein trrestrischer Graphirt, wen ich noch untersucht habe. Die Farbe geht dabei zuerst von Schwarz in Grun über und nach zwei- bis arcimaliger Erneuerung des Oxydationsgemisches hat man eine volikommen weifse Sulistanz, welche gewaschen

*) Ann. chim. pirys. [4] 18, 392; 80, 419. 
und über Schwefelsäure getrocknet ein gelbes, etwas anhängendes Pulver darstellt. Wird dieses von Neuem mit dem Oxydationsgemisch behandelt, so vermindert sich seine Menge und nach vierbis fünfmaliger Erneuerung des 0xydationsgemisches bleibt eine gummiartige Masse, welche am Filter klebt und sich nicht vollständig auswaschen läfst. Nach dem Trocknen hängt sie fest an dem Filter und läfst sich davon nur derart trennen, dafs man das angefeuchtete Papier mit den Fingern abreibt; es bleibt eine zähe häutige Massc.

Von terrestrischen Graphiten habe ich den sibirischen, sowie Cumberland- und Ceylongraphit untersucht. Von diesen unterscheiden sich die meteorischen Graphite, wit schon erwăhnt, durch die raschere Oxydation; sie werden in dem dritten bis fünften Theil der Zuit oxydirt; ferner dadurch, dats das Oxydationsproduct der meteorischen Graphite bei wiederholter Einwirkung des Oxydationsmittels sich vermindert und in seinen Eigenschaften eine viel durchgreifendere Verånderung erfeidet, ähnlich wit bei den nicht eigentlich graphitischen Kohlearten. Diesem Vtrhalten nach stehen also die meteorischen Graphite zwischen den eigentlichen Graphiten und der gemeinen Kohle, den ersteren jedoch viel näher.

Ich gehe nun zur Untersuchung der kohleartigen Substanz der schwarzen Muteorite über. Da ist freilich das Material sehr selten, immerhin genügte, was ich davon in meiner Sammlung hatte, einige vergleichende Versuche anzustellen.

\section{Alais - Meteorit.}

Zwei Gramm der fein gepulverten Substanz wurden mit kochendem $W$ asser ausgezogen und das wieder getrocknete Pulver, wie oben beschrieben, mit Aether behandelt. Beim Verdunsten des Aethers bedeckten sich die Wände des Becherglases mit Krystallnadeln, dazwischen fanden sich auch 
einige rhombenförmige Kryställchen. Der Rũckstand roch ganz so wie das Aetherextract aus dem Seviergraphit und wie bei jenem verlor sich der Geruch bei mehrtägigem Stehen an der Luft; die Krystalle stimmen in Form und Aussehen mit den aus jenem Graphit erhaltenen überein und auch das Verhalten in der Hitze ist das gleiche.

Diese krystallinische Substanz ist schon von Roscoe untersucht worden, so weit es die geringe Menge, die ihm zu Gebote stand, gestattete. Meine Beobachtungen sind vollständig in Uebereinstimmung mit denten Roscoe's; die Gleichartigkeit meiner Krystalle und der aus Graphit erbaltenen ist daher nicht zu bezweifeln.

Ich will nicht unterlassen zu bemerken, dafs Wöhler bei der Untersuchung des Kabameterorits das Vorkommen von Kohlenwasserstoff in den schwarzen Meteoriten zuerst beobachtete.

\section{Orgueil-Meteorit.}

Dieser Meteorit, einer der interessantesten von allen Kohlenmeteoriten, die man kennt, wurde schon von Cloèz *) und von $\mathrm{Pisani}$ *) eingehend untersucht. Cloëz stellt die kohlenstoffhaltige Substanz denı Humus zur Seite; er analysirte sie als Ganzes und fand in der bei $110^{\prime \prime}$ getrockneten Substanz 63,45 Kohlenstoff, 5,98 W usserstoff, 30,75 Sauerstoff.

Diese kohlenstoff haltige Substanz, welche etwa 4 bis 6 pC. des ganzen Meteorits ausmacht, habe ich nicht weiter untersucht, da mein Augenmerk hauptsächlich auf den in Aether und Schwefelkohlenstoff löslichen krystallinischen Körper gerichtet war. Ich fand, dafs der Meteorit davon $1 / 2 \mathrm{pC}$. enthält.

*) Compt. rend. 58, 984 ; Jahresbericht f. Chemie u. s. w. f. 1864, 898.

**) Compt. rend. 50, 132; Jahresbericht f. Chemie u. B. T. f. 1864, 899. 
Das gepulverte Material wurde mit heifsem Wasser möglichst vollständig ausgezogen und sorgfaltig ausgewaschen; was sich löste betrug $8,055 \mathrm{pC}$. des Ganzen. Der in Wasser unlösliche Rückstand wurde nach dem Trocknen wie oben mit Aether behandeit. Beim Verdunsten der ätherischen Lòsung erschienen witder die Krystallnadeln an den Wänden und zahireiche rhombenförnige Krystälichen am Boden des Becherglases. Nach Aussehen und Verhalten beim Erhitzen sind sic identisch mit den Krystallen aus dem Seviergraphit. Die unn folgende Behandlung mit Schwefelkohlenstof ergab beim Verdunsten des Lösungsmittels einen gelhen Hückstand, der wie Scliwefel aussah und sich beim Erhitzen wie Schwefel gemischt mit irgend einer Kohlenstofferbindung, also ganz wie die in gleicher $W$ eise aus dem Seviergraphit erhalsene Substanz verhielt.

Der beim Verdunsten der ätherischen Lösung erhaltene Rückstand wurde, nachdem die Krystallnadeln von der Gefärswand mittelst oines Hornspateis abgekratzt und entfernt worden waren, in Schwefelkohlenstoff gelost. Beim Verdunsten des Lösungsmittels hinterblieb eine feste gelbe Masse, umgelen von einem dunkelbraunen Rıng einer halbflüssigea Masse; letztere, es war nur eune minimale Venge, ist offonbar eine schwefelfreie Kohlenstoffverbindung, während der gelbe Körper aus Schwefel hesteht und nur wenig der Kolilenstoffverbindung enthiah.

Aus 50 Grm. Meteoritsubstanz rhielt ich etwas über $400 \mathrm{Mgrm}$. dieses Gemenges, das jedoch zum weitaus grörsuren Theil aus Schwcfel besteht. Maine Versuche, Schwefel und Kohlenstofferbindung von einander $z$ trennen, waren erfolglos.

Kohlenvasserstoff aus Meteorgraphit und Kohlenmetecriten. Uafs der Kohlenwassersiof in dea Heteuniten bereits entblten war zur Zeit ihres Falles, kann keinem $Z_{w}$ eifel unter- 
liegen. Die Kohlenmeteorite enthalten Nichts, was eine spätere Bildung erklärlich scheinen liefse und was die Graphitkerne anlangt, so waren diese eingebettet im Inneren einer Eisenmasse von mehr als zwanzig Centimeter Durchmesser und überdem war das zur Untersuchung verwendete Pulver aus dem Inneren eines compacten Graphitknollens genommen.

Es hommt mir sehr wahrscheinlich vor, dafs der Kohlenwasserstoff mit dem Schwefel wirklich verbunden ist. Diefs ist zwar nicht chemisch erwiesen, es wird aber wahrscheinlich wegen des eigenthümlichen Geruchs des Aetherextractes: auch spricht sehr dafür ein höchst sonderbares Verhalten des Wasserextractes aus den Orgueilmeteorit, das ich nur kurz andeuten will, indem ich mir weitere Mittheilungen für eine spätere Gelegenheit vorbehalte.

Wenn man eine Probe, sagen wir 2 Gral., ron der gepulverten Substanz des Orgueil-Mettorits nit Wasser übergiefst und in Wassırbad einige Zeit erwärmt, so lafst sich ein besonterer Geruch hicht bemerken; auch wenn man den flurirten wässerigen Auszug auf dem Wasserbad zur Trockne verdampft, ist kein Geruch wahrzunehmen und endlich ist auch der trockene Rückstand getuchlos. Wirt jedoch der Rückstand mit ein wenig Wasser ( $1 / 2$ bis 1 CC.) angefeuchlet unter die Nase gehrachi, so bemerkt man einen ausgesprochen knoblauchartigen Geruch, der manchnal so stark ist, dars er geradezu widerlich wird und an Asa foetida erinnert. 1)ars dieser Geruch ron einer Schwefelverbindung herruhre, wird wohl jeder Chemiker anzunchmen geneigt sein; vieileicht von einer Spur einer Schwefelverbindung ahnlich den Aelhylensulfhydrat $C_{4} H_{6} S_{4}$. Die Krystallnädelchen aus der ätlerischen Lösang mögen in ihrer Zusammensetzung von dem festen Fünfrach-Schwefelathylen $C_{4} H_{6} S_{5}(?)$ nicht sehr entfernt sein. Was ich davon aus dem oberen Theil des Becherglases, in welchem die ätherische Lïsung verdunstete, yon der Wand 
zusammenkratzen konnte, gab bei der Analyse 76,65 Schwefel, 15,00 Kohlenstoff und 3,00 Wasserstoff. Der Formel $C_{4} H_{6} S_{6}$ würde entsprechen 75,00 Schwefel, 20,00 Kohlenstoff, 5,00 Wasserstoff (?). Bei meiner Analyse ist übrigens nur die Schwefelbestimmung ganz verlāssig; die zur Kohlenstoff- und Wasserstoffbestimmung verwendete Menge war zu gering, um verlässige Zahlen liefern.

Roscoe verbrannte 0,008 Grm. der gleichen Substanz aus dem Alais-Meteorit in trockenem Sauerstoffgas und erhielt 0,010 schweflige Sãure, 0,008 Kohlensäure und 0,003 Wasser, was 125 Theilen Schwefel auf 54 Kohlenstoff und 10 Wasserstoff entspricht. Auch diese Zahlen können bei der geringen Menge von Substanz $(0,008 \mathrm{Grm}$.) nur als annähernd gelten; so lange wir jedoch keine besserc Analyse haben, müssen wir uns an diese halten.

Diefs sind im Wesentlichen die Resultate meiner Untersuchungen, vielfach unvollständig aus Mangel an Material sowohl, als aus Mangel an geteigneten Untersuchungsmethoden für so winzige Mengen, entlialten sie doch manches Neue und sind nicht ohne Interesse und Bedeutung. Dafs die Graphitconcretionen im Meteoreisen Körper wie freien Schwefel und Kohlenwasserstoff oder Sulfokohlenwasserstoff enthalten, liefs sich gewifs nicht erwarten, denn Alles weist darauf hin, dafs diese Eisenmassen sich einmal in feurig-flüssigem $\mathrm{Zu}$ stand befunden haben müssen *).

Ueber die Bildung und Zusammenhäufung des Meteor-

*) F. Mohr hat neuerdings (diese Annalen 128,257 ) die Hypothese aufgestellt, dafs die Eisen- und Steinmeteore auf nassem Weg ontatanden seien; was or zur Begrüudung diesor Ansicht beibringt, ist 2 war sehr geistreich, aber nicht entfernt berroisend und zur Erklitung der bekannten Thatsachen augreichend. 
graphits eine plausible Hypothese aufzustellen vermag ich nicht. Während derselbe mit dem Graphit aus Hochofenschlacke oder Gufseisen in Anssehen und Gefüge gar keine Aehnlichkeit hat, verhält er sich doch ganz ähnlich wie dieser gegen chlorsaures Kali und Salpetersauure, andererseits gleicht er in den aufseren Eigenschaften dem amorphen Kohlenstoff aus Gufseisen, von welchem er sich aber durch das Verhalten gegen jenes Oxydationsgemisch bestimmt verschieden erweist. Ich bin daher noch am meisten geneigt, mich der Ansicht Berthelot's anzuschliefsen, dafs der Meteorgraphit durch Einwirkung von Schwefelkohlenstoff auf glühendes Eisen gebildet sein möge; denn bei dieser Reaction entsteht ein dem Meteorgraphit ähnlicher amorpher Graphit; das gleichzeitige Vorkommen von Schwefeleisen, Schwefel und Kohlenwasserstoff oder geschwefeltem Kohlenwasserstoff dürfte dieser Ansicht zur weiteren Stütze gereichen.

Der Kohlenstoff der schwarzen Meteorite, wie Orgueil, Alais u. s. w., muls nach meiner Meinung ähnlichen Ursprungs sein wie der der Eisenmeteorite, denn beide enthalten, wie ich gezeigt habe, den gleichen, in Aether und Schwefelkohlenstoff löslichen krystallinischen Körper. Ihr Verhalten bei der Oxydation ist zwar etwas verschieden, doch mag diefs von einer Verschiedenheit der Reactionsbedingungen bei ihrer Bildung herrühren.

Die kohlenstoff haltige Substanz der schwarzen Meteorite für eine Art Humus zu halten, widerspricht Allem, was wir über Hurnus wissen. Sie ist so gut wie unlöslich in Kaliund Natronlauge, giebt nach dem Trocknen bei $110^{\circ}$ erst in sehr hoher Temperatur Wasser ab und verbrennt auf dem Platinblech leicht und fast ohne Geruch, unter Hinterlassung von viel Asche. Sie beträgt nach meinen Versuchen etwa 41/2 pC. vom Gewicht des ganzen Meteorits. Die Bildung einer entsprechenden Menge von organischer Substanz würde 
eine sehr üppige Vegetation voraussetzen, für diese aber möchten die Mineralbestandtheile der Meteorite einen sehr wenig versprechenden Boden abgeben.

Sehr wahrscheinlich steht die kohlenstof haltige Substanz der schwarzen Meteorite in ihren Eigonschaften dem beim Auflösen von weifsem Gufseisen zurückbleibenden sogenannten Kohlenstoflhydrat nahe, welches zuerst von Eggertz erwäbnt wird. Schülzenberger und Bourgeois *) haben diesen Körper genauer beschrieben; sie geben für seine $\mathrm{Zu-}$ sammensetzung das Verhältnifs $\mathrm{C}_{11}: 3 \mathrm{H}_{2} \mathrm{O}$; dem entspricht 70,96 Kohlenstoff, 3,23 Wasserstoff, 25,91 Sauerstoff, wälırend Glo ëz für die bei $110^{\circ}$ getrocknete kohlenstoff haltige Substanz des Orgueil 63,45 Kohlenstoff, 5,98 Wasserstoff, 30,75 Sauerstoff fand. Zieht man in Betracht, dafs ein Theil des Wasserstoffs als Kolblenwasserstoff vorhanden, so dürfte der Rest zu dem vorhandenen Sauerstulf amnahernd in dem nämlichen Verhältuirs stethen, wie im Wasser.

Vargeblich habe ich versucht, die kohlenstoff haltige Substanz ganz aschenfrei zu bekommen. Durch Flufssäure, allein oder in Verbindung nit Salpetersäure, oder durch eine Mischung von fluornatrium, Schwefelsäure und wenig Wasser und nachfolgende Behandlung mil kalter Salpetersäure gelang es zwar leicht den gröfsten Theil der Mineralbestandtieile auszuziehen, zugleich aber ubten diose Reagentien, selbst wenn Temperaturerböhung sorgfältig vermieden wurde, einen verändernden Einflufs auf die kohlenstoff haltige Substanz selbst. Nach dieser Behandlung bei $110^{\prime \prime}$ getrocknet verlor sie im Röhrchen urhitat bej $250^{\circ}$ Wasser und entwickelte einen sehr starken bituminösen Geruch, vorübergehend dem widerlichen Geruch einer sehr schlechten Cigarre alıniich; denselben Ge-

*) Bull. soc. chim. N. S. 88,387 : 
ruch giebt das sugerannte Kohlenstoflhydrat aus Gufseisen beim Erhitzen.

Nach diesen Versuchen ist also die kohleartige Substanz der Meteorite weder eigentliche Kohle, noch Humus, sondern höchst wahrscheinlich eine dem erwähnten sogenannten Kohlenstoff hydrat ähnliche Verbindung. Aufklärung über die wahre Natur dieser Substanz müssen wir von weiteren Untersuchungen der verschiedenen Verbindungen, welche im Aussehen dem amorphen Kohlenstoff ähnlich sind, wie Hydrographitoxyd und Pyrographitoxyd, Kohlenstoffhydrat und ähnliclien, die noch entdeckt werden nögen, erwarten. Für jetzt sind Bildung und Ursprung dieser Substanz noch eben so in Dunkel gehüllı, wie die Abstanmung der Kōrper, in denen sie sich findel.

Was wir wissen ist, dafs diese kohleartige Substanz von den gleichen Mineralien begleitet wird, welche auch die Hauptbestandtheile aller Steinmeteoriten bilden, sowie von Nickeleistr, welches sowohl in den Stein- wie in den Metallmeteoriten gefunden wird; ferner dafs dicselbe eigenthümliche, in Aether und Schwefelkohlenstoff lösliche krystallinische Körper enthäl, die auch in den Graphitknollen der Eisenmeteorite vorkommen; endlich haben wir in diesen Graphitknollen Magnesia gefunden, diesen nie fehlenden Bestandtheil der Mineralien, welche die Steinmeteorite bilden. 